Mathematical Modelling and Analysis

Volume 16 Number 2, June 2011, 304-314

Doi:10.3846/13926292.2011.580470

(C) Vilnius Gediminas Technical University, 2011
www.tandf.co.uk/journals/TMMA

Publisher: Taylor\&Francis and VGTU

Online ISSN: 1648-3510

Print ISSN: 1392-6292

\title{
Eventual Stability and Eventual Boundedness for Impulsive Differential Equations with "Supremum"
}

\section{Ivanka Stamova}

\author{
Burgas Free University \\ San Stefano St., 8000 Burgas, Bulgaria \\ E-mail(corresp.): stamova@bfu.bg
}

Received August 24, 2010; revised March 9, 2011; published online May 1, 2011

\begin{abstract}
Eventual stability and eventual boundedness for nonlinear impulsive differential equations with supremums are studied. The impulses take place at fixed moments of time. Piecewise continuous Lyapunov functions have been applied. Method of Razumikhin as well as comparison method for scalar impulsive ordinary differential equations have been employed.
\end{abstract}

Keywords: Eventual stability, eventual boundedness, impulsive differential equations, supremum, Lyapunov functions.

AMS Subject Classification: $34 \mathrm{~K} 45$.

\section{Introduction}

The stability of solutions of differential equations via Lyapunov method has been intensively investigated in the past. In many real cases, it is obligatory to study the stability of such sets, which are not invariant with respect to a given system of differential equations. This immediately excludes the stability in the sense of Lyapunov. Examples for that can be found when self-controlled systems of management are being studied [2]. For the problem, arisen in this situation, to be solved, a new notion is introduced - eventual stability $[7,17]$. In this case, the set under consideration, despite not being invariant in the usual sense, is invariant in the asymptotic sense.

Impulsive differential equations are found in almost every domain of applied sciences. Numerous examples were given in Bainov's and his collaborators' book [6]. Some impulsive differential equations have been recently introduced in population dynamics [15], neural networks [12], the chemostat [16], etc. In the mathematical simulation in various important branches of control theory, pharmacokinetics, economics, etc. one has to analyse the influence of both the maximum of the function investigated and its impulsive changes. An adequate 
mathematical apparatus for simulation of such processes are the impulsive differential equations with supremums $[1,4,8]$. To the best of our knowledge, there are no results considering the stability of nonlinear impulsive differential equations with supremums, which is very important in theories and applications and also is a very challenging problem.

In the present paper eventual stability of $x=0$ and eventual boundedness of the solutions with respect to the system of impulsive differential equations with "supremum" is defined. By employing a class of piecewise continuous functions which are generalization of the classical Lyapunov's functions $[6,11]$ coupled with the Razumikhin technique $[3,5,9,10,13,14,15]$ some sufficient conditions are found.

\section{Preliminary Notes and Definitions}

Let $R^{n}$ be the $n$-dimensional Euclidean space with norm |.|; $\Omega$ be a domain in $R^{n}$ containing the origin; $R_{+}=[0, \infty) ; R=(-\infty, \infty) ; t_{0} \in R_{+}, \tau>0$.

Let $J \subseteq R$. Define the following class of functions: $P C[J, \Omega]=\{\sigma: J \rightarrow$ $\Omega: \sigma(t)$ is a piecewise continuous function with points of discontinuity $\tilde{t} \in J$ at which $\sigma(\tilde{t}-0)$ and $\sigma(\tilde{t}+0)$ exist and $\sigma(\tilde{t}-0)=\sigma(\tilde{t})\}$.

Consider the following system of impulsive differential equations with "supremum"

$$
\left\{\begin{array}{l}
\dot{x}(t)=f\left(t, x(t), \sup _{s \in[t-\tau, t]} x(s)\right), \quad t \neq t_{k}, \\
\Delta x\left(t_{k}\right)=I_{k}\left(x\left(t_{k}-0\right)\right), \quad t_{k}>t_{0}, \quad k=1,2, \ldots,
\end{array}\right.
$$

where $f:\left[t_{0}, \infty\right) \times \Omega \times \Omega \rightarrow R^{n} ; I_{k}: \Omega \rightarrow R^{n}, k=1,2, \ldots ; \Delta x(t)=$ $x(t+0)-x(t-0) ; t_{0}<t_{1}<t_{2}<\cdots ; \lim _{k \rightarrow \infty} t_{k}=\infty$. Let $\varphi_{0} \in P C[[-\tau, 0], \Omega]$. Denote by $x(t)=x\left(t ; t_{0}, \varphi_{0}\right), x \in \Omega$ the solution of system (2.1), satisfying the initial conditions:

$$
\left\{\begin{array}{l}
x\left(t ; t_{0}, \varphi_{0}\right)=\varphi_{0}\left(t-t_{0}\right), \quad t_{0}-\tau \leq t \leq t_{0}, \\
x\left(t_{0}+0 ; t_{0}, \varphi_{0}\right)=\varphi_{0}(0)
\end{array}\right.
$$

and by $J^{+}\left(t_{0}, \varphi_{0}\right)$ - the maximal interval of type $\left[t_{0}, \beta\right)$ in which the solution $x\left(t ; t_{0}, \varphi_{0}\right)$ is defined.

The solution $x\left(t ; t_{0}, \varphi_{0}\right)$ of problem $(2.1),(2.2)$ is a piecewise continuous function in interval $J^{+}\left(t_{0}, \varphi_{0}\right)$ with points of discontinuity of the first kind $t=t_{k}, k=1,2, \ldots$ at which it is continuous from the left, i.e. the following relations are satisfied:

$$
x\left(t_{k}-0\right)=x\left(t_{k}\right), \quad x\left(t_{k}+0\right)=x\left(t_{k}\right)+I_{k}\left(x\left(t_{k}\right)\right), \quad t_{k} \in J^{+}\left(t_{0}, \varphi_{0}\right) .
$$

Introduce the following notations:

$$
\begin{aligned}
& G_{k}=\left\{(t, x) \in\left[t_{0}, \infty\right) \times \Omega: t_{k-1}<t<t_{k}\right\}, k=1,2, \ldots ; G=\bigcup_{k=1}^{\infty} G_{k} ; \\
& \|\phi\|=\sup _{t \in\left[t_{0}-\tau, t_{0}\right]}\left|\phi\left(t-t_{0}\right)\right| \text { is the norm of the function } \phi \in P C[[-\tau, 0], \Omega] ; \\
& S_{\rho}=\left\{x \in R^{n}:|x|<\rho\right\}, \rho=\text { const }>0 ;
\end{aligned}
$$


$K=\left\{a \in C\left[R_{+}, R_{+}\right]: a(u)\right.$ is strictly increasing and such that $\left.a(0)=0\right\}$.

Introduce the following conditions:

H2.1. $f \in C\left[\left[t_{0}, \infty\right) \times \Omega \times \Omega, R^{n}\right]$.

H2.2. $f(t, 0,0)=0, t \in\left[t_{0}, \infty\right)$.

H2.3. The function $f$ is Lipschitz continuous with respect to its second and third arguments in $\left[t_{0}, \infty\right) \times \Omega \times \Omega$, uniformly on $t \in\left[t_{0}, \infty\right)$.

H2.4. $I_{k} \in C\left[\Omega, R^{n}\right], k=1,2, \ldots$

H2.5. $I_{k}(0)=0, k=1,2, \ldots$

H2.6. The functions $\left(I+I_{k}\right): \Omega \rightarrow \Omega, k=1,2, \ldots$ where $I$ is the identity in $\Omega$.

H2.7. $t_{0}<t_{1}<t_{2}<\cdots$.

H2.8. $\lim _{k \rightarrow \infty} t_{k}=\infty$.

In the further considerations we shall use the class $V_{0}$ of piecewise continuous auxiliary functions $V:\left[t_{0}, \infty\right) \times \Omega \rightarrow R_{+}$which are analogues of Lyapunov's functions [11].

Definition 1. A function $V:\left[t_{0}, \infty\right) \times \Omega \rightarrow R_{+}$belongs to class $V_{0}$, if:

1. $V$ is continuous in $G$, locally Lipschitz continuous with respect to its second argument on each of the sets $G_{k}, k=1,2, \ldots$ and $V(t, 0)=0$, $t \in\left[t_{0}, \infty\right)$.

2. For each $k=1,2, \ldots$ and $x \in \Omega$

$$
V\left(t_{k}-0, x\right)=V\left(t_{k}, x\right) \text { and } V\left(t_{k}+0, x\right)=\lim _{\substack{t \rightarrow t_{k} \\ t>t_{k}}} V(t, x) \text { exists. }
$$

Definition 2. Given a function $V \in V_{0}$. For any $t \geq t_{0}, t \neq t_{k}, k=1,2, \ldots$ and any function $\phi \in P C[[t-\tau, t], \Omega]$ the upper right-hand derivative of $V$ with respect to system (2.1) is defined by

$$
\begin{gathered}
D^{+} V(t, \phi)=\lim _{h \rightarrow 0^{+}} \sup \frac{1}{h}[V(t+h, \phi(t)+h f(t, \phi(t), \\
\left.\left.\left.\sup _{s \in[-\tau, 0]} \phi(t+s)\right)\right)-V(t, \phi(t))\right] .
\end{gathered}
$$

Note that in Definition 2, $D^{+} V(t, \phi)$ is a functional whereas $V$ is a function.

Introduce the set $P C^{1}\left[J, R^{n}\right]=\left\{\sigma \in P C\left[J, R^{n}\right]: \sigma(t)\right.$ is continuously differentiable everywhere except some points $t_{k}$ at which $\dot{\sigma}\left(t_{k}-0\right)$ and $\dot{\sigma}\left(t_{k}+0\right)$ exist and $\left.\dot{\sigma}\left(t_{k}-0\right)=\dot{\sigma}\left(t_{k}\right), \quad k=1,2, \ldots\right\}$. We remark that if $V \in V_{0}$ and $V \in P C^{1}\left[\left[t_{0}, \infty\right) \times \Omega, R_{+}\right]$, then $(2.3)$ reduces to

$$
D^{+} V(t, \phi)=\frac{\partial V(t, \phi(t))}{\partial t}+\Delta_{x} V(t, \phi(t)) . f\left(t, \phi(t), \sup _{s \in[-\tau, 0]} \phi(t+s)\right) .
$$


Together with system (2.1), we shall consider the comparison equation

$$
\left\{\begin{array}{l}
\dot{u}(t)=g(t, u), \quad t \geq t_{0}, \quad t \neq t_{k}, \\
\Delta u\left(t_{k}\right)=B_{k}\left(u\left(t_{k}\right)\right), \quad t_{k}>t_{0}, k=1,2, \ldots,
\end{array}\right.
$$

where $g:\left[t_{0}, \infty\right) \times R_{+} \rightarrow R_{+}, B_{k}: R_{+} \rightarrow R, k=1,2, \ldots$

In the proof of the main results we shall use the following lemmas:

Lemma 1. Let the conditions H2.1, H2.3, H2.4, H2.6, H2.7 and H2.8 hold. Then $J^{+}\left(t_{0}, \varphi_{0}\right)=\left[t_{0}, \infty\right)$.

Proof. Since the conditions H2.1, H2.3, H2.4 and H2.6 hold then from the existence theorem for the equation $\dot{x}(t)=f\left(t, x(t), \max _{s \in[t-\tau, t]} x(s)\right)$ without impulses [3] it follows that the solution $x(t)=x\left(t ; t_{0}, \varphi_{0}\right)$ of the problem (2.1), $(2.2)$ is defined on each of the intervals $\left(t_{k-1}, t_{k}\right], k=1,2, \ldots$ From conditions H2.7 and H2.8 we conclude that it can be prolonged continuously for $t \geq t_{0}$.

Let us note that the problems of existence, uniqueness, and continuability of the solutions of functional differential equations without impulses has been investigated in the monograph [3].

Lemma 2. Assume that:

1. Conditions H2.1, H2.3, H2.4, H2.6, H2.7 and H2.8 hold.

2. The function $g:\left[t_{0}, \infty\right) \times R_{+} \rightarrow R_{+}$is continuous in each of the sets $\left(t_{k-1}, t_{k}\right] \times R_{+}, k=1,2, \ldots$

3. $B_{k} \in C\left[R_{+}, R\right]$, and $\psi_{k}(u)=u+B_{k}(u) \geq 0, k=1,2, \ldots$ are nondecreasing with respect to $u$.

4. The maximal solution $u^{+}\left(t ; t_{0}, u_{0}\right)$ of the scalar equation (2.4) with $u^{+}\left(t_{0}+0 ; t_{0}, u_{0}\right)=u_{0}, u_{0} \in R_{+}$is defined in the interval $\left[t_{0}, \infty\right)$.

5. The function $V \in V_{0}$ is such that $V\left(t_{0}+0, \varphi_{0}(0)\right) \leq u_{0}$,

$$
V\left(t+0, x+I_{k}(x)\right) \leq \psi_{k}(V(t, x)), \quad x \in \Omega, t=t_{k}, k=1,2, \ldots,
$$

and the inequality $D^{+} V(t, \phi(t)) \leq g(t, V(t, \phi(t))), t \neq t_{k}, k=1,2, \ldots$ is valid for any $t \in\left[t_{0}, \infty\right)$ and any $\phi \in P C[[t-\tau, t], \Omega]$ such that $V(t+$ $s, \phi(t+s))<V(t, \phi(t)), s \in[-\tau, 0)$.

Then

$$
V\left(t, x\left(t ; t_{0}, \varphi_{0}\right)\right) \leq u^{+}\left(t ; t_{0}, u_{0}\right), \quad t \in\left[t_{0}, \infty\right) .
$$

Proof. From Lemma 1 it follows that $J^{+}\left(t_{0}, \varphi_{0}\right)=\left[t_{0}, \infty\right)$ and the solution $x=x\left(t ; t_{0}, \varphi_{0}\right)$ of the problem $(2.1),(2.2)$ is such that

$$
x \in P C\left[\left(t_{0}-\tau, \infty\right), \Omega\right] \cap P C^{1}\left[\left[t_{0}, \infty\right), \Omega\right] .
$$


The maximal solution $u^{+}\left(t ; t_{0}, u_{0}\right)$ of the system $(2.4)$ is defined by the equality

$$
u^{+}\left(t ; t_{0}, u_{0}\right)= \begin{cases}r_{0}\left(t ; t_{0}, u_{0}^{+}\right), & t_{0}<t \leq t_{1}, \\ r_{1}\left(t ; t_{1}, u_{1}^{+}\right), & t_{1}<t \leq t_{2}, \\ \ldots & \\ r_{k}\left(t ; t_{k}, u_{k}^{+}\right), & t_{k}<t \leq t_{k+1}, \\ \ldots, & \end{cases}
$$

where $r_{k}\left(t ; t_{k}, u_{k}^{+}\right)$is the maximal solution of the system without impulses $\dot{u}=F(t, u)=f\left(t, u, \max _{s \in[t-\tau, t]} u(s)\right)$ in the interval $\left(t_{k}, t_{k+1}\right], k=0,1,2, \ldots$, for which $u_{k}^{+}=\psi_{k}\left(r_{k-1}\left(t_{k} ; t_{k-1}, u_{k-1}^{+}\right)\right), k=1,2, \ldots$ and $u_{0}^{+}=u_{0}$.

Let $t \in\left(t_{0}, t_{1}\right]$. Then, from the corresponding comparison theorem for the continuous case [7], it follows that $V\left(t, x\left(t ; t_{0}, \varphi_{0}\right)\right) \leq u^{+}\left(t ; t_{0}, u_{0}\right)$, i.e. the inequality $(2.5)$ is valid for $t \in\left(t_{0}, t_{1}\right]$. Suppose that $(2.5)$ is satisfied for $t \in\left(t_{k-1}, t_{k}\right], k>1$. Then, using condition 5 of Lemma 2 and the fact that the function $\psi_{k}$ is non-decreasing, we obtain

$$
\begin{aligned}
& V\left(t_{k}+0, x\left(t_{k}+0 ; t_{0}, \varphi_{0}\right)\right) \leq \psi_{k}\left(V\left(t_{k}, x\left(t_{k} ; t_{0}, \varphi_{0}\right)\right)\right) \\
& \quad \leq \psi_{k}\left(u^{+}\left(t_{k} ; t_{0}, \varphi_{0}\right)\right)=\psi_{k}\left(r_{k-1}\left(t_{k} ; t_{k-1}, u_{k-1}^{+}\right)\right)=u_{k}^{+} .
\end{aligned}
$$

We apply again the comparison theorem for the continuous case in the interval $\left(t_{k}, t_{k+1}\right]$ and obtain $V\left(t, x\left(t ; t_{0}, \varphi_{0}\right)\right) \leq r_{k}\left(t ; t_{k}, u_{k}^{+}\right)=u^{+}\left(t ; t_{0}, u_{0}\right)$, i.e. the inequality (2.5) is valid for $t \in\left(t_{k}, t_{k+1}\right]$.

\section{Main Results}

\subsection{Eventual stability}

We shall also use the following notations:

$$
\begin{aligned}
& B_{\alpha}=\left\{(t, x) \in\left[t_{0}, \infty\right) \times R^{n}:|x|<\alpha\right\} ; \\
& \bar{B}_{\alpha}=\left\{(t, x) \in\left[t_{0}, \infty\right) \times R^{n}:|x| \leq \alpha\right\}, \quad \alpha>0 .
\end{aligned}
$$

We shall use the following definitions of eventual stability of $x=0$ for the system (2.1).

Definition 3. The set $x(t) \equiv 0$ is said to be:

(a) eventually stable set of system (2.1), if

$$
\begin{aligned}
& (\forall \varepsilon>0)(\exists T=T(\varepsilon)>0)\left(\forall t_{0} \geq T\right)\left(\exists \delta=\delta\left(t_{0}, \varepsilon\right)>0\right) \\
& \left(\forall \varphi_{0} \in P C[[-\tau, 0], \Omega]:\left\|\varphi_{0}\right\|<\delta\right)\left(\forall t \geq t_{0}\right):\left|x\left(t ; t_{0}, \varphi_{0}\right)\right|<\varepsilon ;
\end{aligned}
$$

(b) uniformly eventually stable set of system (2.1), if the number $\delta$ in (a) is independent of $t_{0} \in R$.

Theorem 1. Assume that:

1. Conditions H2.1-H2.8 hold. 
2. $g(t, 0)=0, t \in\left[t_{0}, \infty\right)$.

3. $B_{k}(0)=0, k=1,2, \ldots$

4. The conditions of Lemma 2 hold, and there exists a function $a \in K$ such that

$$
a(|x|) \leq V(t, x), \quad a \in K,(t, x) \in\left[t_{0}, \infty\right) \times \Omega,
$$

where $V \in V_{0}$.

Then if the set $u=0$ is an eventually stable set of system (2.4), then the set $x=0$ is an eventually stable set of system (2.1).

Proof. Let $\varepsilon>0$ be such that $S_{\varepsilon} \subset \Omega$ and the set $u=0$ be an eventually stable of system (2.4). Then there exist $T>0$ and $\delta_{1}>0$ such that

$$
u_{0}<\delta_{1} \quad \text { implies } u^{+}\left(t ; t_{0}, u_{0}\right)<a(\varepsilon), \quad t \geq t_{0}
$$

for some given $t_{0} \geq T$, where the maximal solution $u^{+}\left(t ; t_{0}, u_{0}\right)$ of $(2.4)$ is defined in the interval $\left[t_{0}, \infty\right)$.

From the properties of the function $V$, it follows that there exists a constant $\delta=\delta\left(t_{0}, \varepsilon\right)>0$ such that if $\left(t_{0}+0, x\right) \in B_{\delta}$, then $V\left(t_{0}+0, x\right) \in S_{\delta_{1}}$. Let $\varphi_{0} \in$ $P C[[-\tau, 0], \Omega]:\left\|\varphi_{0}\right\|<\delta$ and $x(t)=x\left(t ; t_{0}, \varphi_{0}\right)$ be the solution of problem $(2.1)$, $(2.2)$. Then $\left|\varphi_{0}(0)\right| \leq\left\|\varphi_{0}\right\|<\delta,\left(t_{0}+0, \varphi_{0}(0)\right) \in B_{\delta}$, hence $V\left(t_{0}+0, \varphi_{0}(0)\right) \in$ $S_{\delta_{1}}$. Thus

$$
u^{+}\left(t ; t_{0}, V\left(t_{0}+0, \varphi_{0}(0)\right)\right)<a(\varepsilon) .
$$

Setting $u_{0}=V\left(t_{0}+0, \varphi_{0}(0)\right)$, we get by Lemma 2 ,

$$
V\left(t, x\left(t ; t_{0}, \varphi_{0}\right)\right) \leq u^{+}\left(t ; t_{0}, V\left(t_{0}+0, \varphi_{0}(0)\right)\right) \quad \text { for } t \geq t_{0} .
$$

Consequently, from (3.1), (3.3) and (3.2), we obtain

$a\left(\left|x\left(t ; t_{0}, \varphi_{0}\right)\right|\right) \leq V\left(t, x\left(t ; t_{0}, \varphi_{0}\right)\right) \leq u^{+}\left(t ; t_{0}, V\left(t_{0}+0, \varphi_{0}(0)\right)<a(\varepsilon), \quad t \geq t_{0}\right.$.

Hence, $\left|x\left(t ; t_{0}, \varphi_{0}\right)\right|<\varepsilon, t \geq t_{0}$ for the given $t_{0}>T$, which proves the eventual stability of the set $x=0$ of (2.1).

Theorem 2. Let the conditions of Theorem 1 hold, and a function $b \in K$ exists such that

$$
V(t, x) \leq b(|x|), \quad(t, x) \in\left[t_{0}, \infty\right) \times \Omega .
$$

Then if the set $u=0$ is an uniformly eventually stable set of system (2.4), then the set $x=0$ is an uniformly eventually stable set of system (2.1).

Proof. Let $\varepsilon>0$ be such that $S_{\varepsilon} \subset \Omega$. Suppose now, that the set $u=0$ be an uniformly eventually stable of system (2.4). Therefore, we have that

$$
u_{0}<\delta_{1} \quad \text { implies } u^{+}\left(t ; t_{0}, u_{0}\right)<a(\varepsilon), \quad t \geq t_{0}
$$

for every $t_{0}>T$ and $\delta_{1}>0$ independent of $t_{0} \in R$. Let $\delta>0$ be such that $\delta<b^{-1}\left(\delta_{1}\right)$ and

$$
\left\|\varphi_{0}\right\|<\delta
$$


Then, from (3.4) and (3.6), it follows

$$
V\left(t_{0}+0, \varphi_{0}(0)\right) \leq b\left(\left|\varphi_{0}(0)\right|\right) \leq b\left(\left\|\varphi_{0}\right\|\right)<b(\delta)<\delta_{1}
$$

which due to (3.5) implies

$$
u^{+}\left(t ; t_{0}, V\left(t_{0}+0, \varphi_{0}(0)\right)\right)<a(\varepsilon), \quad t \geq t_{0} .
$$

We claim that $\left\|\varphi_{0}\right\|<\delta$ implies $\left|x\left(t ; t_{0}, \varphi_{0}\right)\right|<\varepsilon, t \geq t_{0}$ for every $t_{0}>T$. If the claim is not true, there exists $t_{0}>T$, a corresponding solution $x\left(t ; t_{0}, \varphi_{0}\right)$ of (2.1) with $\left\|\varphi_{0}\right\|<\delta$, and $t^{*}>t_{0}$ such that,

$$
\left|x\left(t^{*} ; t_{0}, \varphi_{0}\right)\right| \geq \varepsilon, \quad\left|x\left(t ; t_{0}, \varphi_{0}\right)\right|<\varepsilon, \quad t_{0} \leq t \leq t_{k},
$$

where $t^{*} \in\left(t_{k}, t_{k+1}\right]$ for some $k$. Then, due to H2.6 and condition 5 of Lemma 2, we can find $t^{0} \in\left(t_{k}, t^{*}\right)$ such that

$$
\left|x\left(t^{0} ; t_{0}, \varphi_{0}\right)\right| \geq \varepsilon \quad \text { and } \quad x\left(t^{0} ; t_{0}, \varphi_{0}\right) \in \Omega .
$$

Hence, setting $u_{0}=V\left(t_{0}, \varphi_{0}\left(t^{0}-t_{k}\right)\right)$, since all the conditions of Lemma 2 are satisfied, we get

$$
V\left(t, x\left(t ; t_{0}, \varphi_{0}\right)\right) \leq u^{+}\left(t ; t_{0}, V\left(t_{0}, \varphi_{0}\left(t^{0}-t_{k}\right)\right)\right) \text { for } t_{0} \leq t \leq t^{0} .
$$

From $(3.8),(3.1),(3.9)$ and $(3.7)$, it follows that

$$
\begin{aligned}
a(\varepsilon) & \leq a\left(\left|x\left(t^{0} ; t_{0}, \varphi_{0}\right)\right|\right) \leq V\left(t^{0}, x\left(t ; t_{0}, \varphi_{0}\right)\right) \\
& \leq u^{+}\left(t^{0} ; t_{0}, V\left(t_{0}, \varphi_{0}\left(t^{0}-t_{k}\right)\right)\right)<a(\varepsilon) .
\end{aligned}
$$

The contradiction obtained proves that (2.1) is uniformly practically stable.

Remark 1. We have assumed in Theorems 1 and 2 stronger requirements on $V$ only to unify all the stability criteria for the comparison equation and for the system under consideration. This obviously puts burden on the comparison equation (2.4). However, to obtain only non-uniform stability criteria, we could weaken certain assumption, as it is stated in the next result.

Theorem 3. Assume that:

1. Conditions $\mathrm{H} 2.1-\mathrm{H} 2.8$ hold.

2. There exists a function $V \in V_{0}$ such that (3.1) holds,

$$
V\left(t+0, x+I_{k}(x)\right) \leq V(t, x), \quad x \in \Omega, t=t_{k}, k=1,2, \ldots,
$$

and the inequality

$$
D^{+} V(t, \phi(t)) \leq p(t) q(t, \phi(t)), \quad t \neq t_{k}, k=1,2, \ldots
$$

is valid for any $t \in\left[t_{0}, \infty\right)$ and any $\phi \in P C[[t-\tau, t], \Omega]$ such that $V(t+$ $s, \phi(t+s))<V(t, \phi(t)), s \in[-\tau, 0), p:\left[t_{0}, \infty\right) \rightarrow R, q:\left[t_{0}, \infty\right) \times \Omega \rightarrow R$. 
3. There exists a number $\Gamma>0$ such that $|q(t, x)| \leq \Gamma,(t, x) \in\left[t_{0}, \infty\right) \times \Omega$.

4. $\int_{t_{0}}^{\infty}|p(t)| d t<\infty$.

Then the set $x=0$ is an eventually stable set of system (2.1).

Proof. Let $\varepsilon>0$ be such that $S_{\varepsilon} \subset \Omega$ and $\Gamma>0$. Let the number $T=T(\varepsilon)>$ 0 be chosen so that for $t \geq T$

$$
\int_{t}^{\infty}|p(s)| d s<\frac{a(\varepsilon)}{2 \Gamma} .
$$

(This is possible in view of condition 4 of Theorem 3.)

Let $t_{0} \geq T$. From the properties of the function $V$, it follows that there exists a constant $\delta=\delta\left(t_{0}, \varepsilon\right)>0$ such that if $\left(t_{0}+0, x\right) \in B_{\delta}$, then $V\left(t_{0}+0, x\right)<$ $\frac{1}{2} a(\varepsilon)$. Let $\varphi_{0} \in P C[[-\tau, 0], \Omega]:\left\|\varphi_{0}\right\|<\delta$ and $x(t)=x\left(t ; t_{0}, \varphi_{0}\right)$ be the solution of problem (2.1), (2.2). Then $\left|\varphi_{0}(0)\right| \leq\left\|\varphi_{0}\right\|<\delta,\left(t_{0}+0, \varphi_{0}(0)\right) \in B_{\delta}$, hence

$$
V\left(t_{0}+0, \varphi_{0}(0)\right)<\frac{1}{2} a(\varepsilon) .
$$

From condition 3 of Theorem 3, (3.11) and (3.12), we have

$$
\int_{t_{0}}^{t} D^{+} V(s, x(s)) d s \leq \Gamma \int_{t_{0}}^{t}|p(s)| d s<\frac{1}{2} a(\varepsilon), \quad t \geq t_{0} .
$$

Let $t_{k+l}<t<t_{k+l+1}$. Then, we have

$$
\begin{aligned}
\int_{t_{0}}^{t} D^{+} V(s, x(s)) d s=\int_{t_{0}}^{t_{1}} D^{+} V(s, x(s)) d s+\sum_{j=2}^{k+l} \int_{t_{j-1}}^{t_{j}} D^{+} V(s, x(s)) d s \\
\quad+\int_{t_{k+l}}^{t} D^{+} V(s, x(s)) d s=V\left(t_{1}, x\left(t_{1}\right)\right)-V\left(t_{0}+0, \varphi_{0}(0)\right) \\
\quad+\sum_{j=2}^{k+l}\left[V\left(t_{j}, x\left(t_{j}\right)\right)-V\left(t_{j-1}+0, x\left(t_{j-1}+0\right)\right]+V(t, x(t))\right. \\
\quad-V\left(t_{k+l}+0, x\left(t_{k+l}+0\right)\right] \geq V(t, x(t))-V\left(t_{0}+0, \varphi_{0}(0)\right) .
\end{aligned}
$$

From (3.1), (3.12)-(3.15), we obtain

$$
\begin{aligned}
a\left(\left|x\left(t ; t_{0}, \varphi_{0}\right)\right|\right) & \leq V\left(t_{0}+0, \varphi_{0}(0)\right)+\int_{t_{0}}^{t} D^{+} V(s, x(s)) d s \\
& <V\left(t_{0}+0, \varphi_{0}(0)\right)+\frac{1}{2} a(\varepsilon)<a(\varepsilon) .
\end{aligned}
$$

Therefore, $\left|x\left(t ; t_{0}, \varphi_{0}\right)\right|<\varepsilon$ for $t \geq t_{0}$.

Remark 2. It is well known that, in the stability theory of functional differential equations, the condition $D^{+} V(t, x(t)) \leq p(t) q(t, x(t))$ allows the derivative of the Lyapunov function to be positive which may not even guarantee the stability of a functional differential system (see $[3,5]$ ). However, as we can see from Theorem 3, impulses have played an important role in stabilizing a functional differential system [14]. 
Theorem 4. Let the conditions of Theorem 3 hold, and a function $b \in K$ exists such that

$$
V(t, x) \leq b(|x|), \quad(t, x) \in\left[t_{0}, \infty\right) \times \Omega .
$$

Then the set $x=0$ is an uniformly eventually stable set of system (2.1).

Proof. Let $\varepsilon>0$ be given. Choose $\delta=\delta(\varepsilon)<b^{-1}\left(\frac{1}{2} a(\varepsilon)\right), 0<\delta<\varepsilon$ and $\Gamma=\Gamma(\varepsilon)>0$ so that $|q(t, x)| \leq \Gamma$ for $(t, x) \in \bar{B}_{\delta}$. Let the number $T=T(\varepsilon)>0$ be chosen so that

$$
\int_{t}^{\infty}|p(s)| d s<\frac{b(\delta)}{\Gamma}, \quad t \geq T .
$$

Let $t_{0} \geq T, \varphi_{0} \in P C[[-\tau, 0], \Omega]:\left\|\varphi_{0}\right\|<\delta$ and let $x(t)=x\left(t ; t_{0}, \varphi_{0}\right)$ be the solution of problem (2.1), (2.2). From (3.1), (3.10), (3.11), (3.16)) and (3.17), we have

$$
\begin{aligned}
a\left(\left|x\left(t ; t_{0}, \varphi_{0}\right)\right|\right) & \leq V\left(t_{0}+0, \varphi_{0}(0)\right)+\int_{t_{0}}^{t} D^{+} V(s, x(s)) d s \\
& \leq b\left(\left|\varphi_{0}(0)\right|\right)+\Gamma \int_{t_{0}}^{t}|p(s)| d s<2 b(\delta)<a(\varepsilon)
\end{aligned}
$$

for $t \geq t_{0}$. Therefore, $\left|x\left(t ; t_{0}, \varphi_{0}\right)\right|<\varepsilon$ for $t \geq t_{0}$.

Example 1. Consider the equation

$$
\left\{\begin{array}{l}
\dot{x}(t)=p(t) \sup _{s \in[t-\tau, t]} x(s), \quad t \neq t_{k}, \\
\Delta x\left(t_{k}\right)=c_{k}, \quad t_{k}>0, k=1,2, \ldots,
\end{array}\right.
$$

where $t \geq 0 ; x \in R_{+} ; \tau>0 ; p \in C\left[R_{+}, R\right] ; c_{k}<0$ and $\left|c_{k}+x\right|<|x|$ for $k=1,2, \ldots, 0<t_{1}<t_{2}<\cdots$ and $\lim _{k \rightarrow \infty} t_{k}=\infty$.

The set $x=0$ is not stable in the sense of Lyapunov, because it is not an equilibrium for the equation (3.18).

Let $\alpha>0$. Consider the function $V(t, x)=|x|$. For $t \geq 0, t \neq t_{k}$, and for any $\phi \in P C[[t-\tau, t], R]$ such that $V(t+s, \phi(t+s))<V(t, \phi(t)), s \in[-r, 0)$, we have

$$
D^{+} V(t, \phi(t))=\operatorname{sign}(\phi(t))\left[p(t) \sup _{s \in[t-\tau, t]} \phi(s)\right] \leq\left|p(t) \| \sup _{s \in[t-\tau, t]} \phi(s)\right| \leq|p(t)||\phi(t)|
$$

for $\phi \in S_{\alpha}$. Also, for $t=t_{k}, k=1,2, \ldots$, we obtain

$$
V\left(t+0, x(t)+c_{k}\right)=\left|c_{k}+x(t)\right| \leq V(t, x(t)) .
$$

If $\int_{0}^{\infty}|p(t)| d t<\infty$, then all conditions of Theorem 3 are satisfied, and the set $x=0$ is an eventually stable set with respect to (3.18). 


\subsection{Eventual boundedness}

In this part of Section 3, we shall apply the direct method of Lyapunov for investigation of eventual boundedness of the solutions of system of the type (2.1) for $\Omega \equiv R^{n}$, i.e. we shall consider the system

$$
\left\{\begin{array}{l}
\dot{x}(t)=f\left(t, x(t), \sup _{s \in[t-\tau, t]} x(s)\right), \quad t \neq t_{k}, \\
\Delta x\left(t_{k}\right)=I_{k}\left(x\left(t_{k}\right)\right), \quad k=1,2, \ldots
\end{array}\right.
$$

where $f:\left[t_{0}, \infty\right) \times R^{n} \times R^{n} \rightarrow R^{n} ; I_{k}: R^{n} \rightarrow R^{n}, k=1,2, \ldots ; \Delta x(t)=$ $x(t+0)-x(t-0) ; t_{0}<t_{1}<t_{2}<\cdots ; \lim _{k \rightarrow \infty} t_{k}=\infty$.

Let $\varphi_{0} \in P C\left[[-\tau, 0], R^{n}\right]$. Denote by $x(t)=x\left(t ; t_{0}, \varphi_{0}\right)$ the solution of (3.19), satisfying the initial conditions

$$
\left\{\begin{array}{l}
x\left(t ; t_{0}, \varphi_{0}\right)=\varphi_{0}\left(t-t_{0}\right), \quad t_{0}-\tau \leq t \leq t_{0}, \\
x\left(t_{0}+0 ; t_{0}, \varphi_{0}\right)=\varphi_{0}(0) .
\end{array}\right.
$$

Definition 4. The solutions of (3.19) are said to be:

(a) eventually equi-bounded, if

$$
\begin{aligned}
& (\forall \alpha>0)(\exists T=T(\alpha)>0)\left(\forall t_{0} \geq T\right)\left(\exists \beta=\beta\left(t_{0}, \alpha\right)>0\right) \\
& \left(\forall \varphi_{0} \in P C\left[[-\tau, 0], R^{n}\right]:\left\|\varphi_{0}\right\|<\alpha\right)\left(\forall t \geq t_{0}\right):\left|x\left(t ; t_{0}, \varphi_{0}\right)\right|<\beta ;
\end{aligned}
$$

(b) uniformly eventually bounded, if the number $\beta$ in (a) is independent of $t_{0} \in R$.

The proofs of the next theorems are similar to the proofs of Theorems 3 and 4. Piecewise continuous Lyapunov functions $V:\left[t_{0}, \infty\right) \times R^{n} \rightarrow R_{+}, V \in V_{0}$ are used.

Theorem 5. Let the conditions of Theorem 3 hold for $\Omega \equiv R^{n}$, and $a(u) \rightarrow \infty$ as $u \rightarrow \infty$. Then the solutions of system (3.19) are eventually equi-bounded.

Theorem 6. Let the conditions of Theorem 5 hold, and a function $b \in K$ exists such that $V(t, x) \leq b(|x|),(t, x) \in\left[t_{0}, \infty\right) \times R^{n}$. Then the solutions of system (3.19) are uniformly eventually bounded.

\section{References}

[1] D. Bainov, Yu. Domshlak and S. Milusheva. Partial averaging for impulsive differential equations with supremum. Georgian Math. J., 3(1):11-26, 1996. Doi:10.1007/BF02256795.

[2] J.R. Haddock. Some new results on stability and convergence of solutions of ordinary and functional differential equations. Funkcial. Ekvac., 19:247-269, 1976.

[3] J. Hale. Theory of Functional Differential Equations. Springer-Verlag, New York, Heidelberg, Berlin, 1977. 
[4] Z. He, P. Wang and W. Ge. Periodic boundary value problem for first order impulsive differential equations with supremum. Indian J. Pure Appl. Math., 34(1):133-143, 2003.

[5] J. Kato. On Liapunov-Razumikhin type theorems for functional differential equations. Funkcial. Ekvac., 16:225-239, 1973.

[6] V. Lakshmikantham, D.D. Bainov and P.S. Simeonov. Theory of Impulsive Differential Equations. World Scientific, Singapore, New Jersey, London, 1989.

[7] V. Lakshmikantham, S. Leela and A. Martynyuk. Practical Stability Analysis of Nonlinear Systems. World Scientific Publishers, Singapore, New Jersey, London, Hong Kong, 1990.

[8] S. Milusheva and D. Bainov. Averaging method for neutral type impulsive differential equations with supremums. Ann. Fac. Sci. Toulouse, 12(3):391-403, 1991.

[9] B.S. Razumikhin. Stability of Hereditary Systems. Nauka, Moscow, 1988. (in Russian)

[10] J. Shen and J. Yan. Razumikhin type stability theorems for impulsive functional differential equations. Nonlinear Anal., 33(5):519-531, 1998. Doi:10.1016/S0362-546X(97)00565-8.

[11] P. Simeonov and D. Bainov. Stability with respect to part of the variables in systems with impulse effect. J. Math. Anal. Appl., 117(1):247-263, 1986. Doi:10.1016/0022-247X(86)90259-3.

[12] G. Stamov. Almost periodic models of impulsive hopfield neural network. $J$. Math. Kyoto Univ., 49(1):57-67, 2009.

[13] I. Stamova. Vector Lyapunov functions for practical stability of nonlinear impulsive functional differential equations. J. Math. Anal. Appl., 325(1):612-623, 2007. Doi:10.1016/j.jmaa.2006.02.019.

[14] I. Stamova. Boundedness of impulsive functional differential equations with variable impulsive perturbations. Bull. Austral. Math. Soc., 77(2):331-345, 2008. Doi:10.1017/S0004972708000439.

[15] I. Stamova and G. Stamov. Lyapunov-Razumikhin method for impulsive functional differential equations and applications to the population dynamics. J. Comput. Appl. Math., 130(1-2):163-171, 2001. Doi:10.1016/S0377-0427(99)00385-4.

[16] S. Sun and L. Chen. Dynamic behaviors of Monod type chemostat model with impulsive perturbation on the nutrient concentration. J. Math. Chem., 42(4):837848, 2007. Doi:10.1007/s10910-006-9144-3.

[17] T. Yoshizawa. Stability Theory by Lyapunov's Second Method. The Mathematical Society of Japan, 1966. 